\title{
Acceso lateroungueal en los tumores glómicos periungueales. Revisión de 75 casos
}

\author{
A. Marcos $^{(1)}$, J. Medina ${ }^{(2)}$, G. Muratore ${ }^{(1)}$, F. Martín ${ }^{(1)}$, L. Reboso $^{(3)}$, \\ G. FOUCHER ${ }^{(4)}$ \\ ${ }^{(1)}$ Médico adjunto. Unidad de Mano, Miembro Superior y Nervio Periférico. Hospital \\ UNIVERSITARIO INSULAR DE GRAN CANARIA \\ ${ }^{(2)}$ Médico adjunto. Coordinador Unidad de Mano, Miembro Superior y Nervio Periférico. \\ HOSPITAL UNIVERSITARIO INSULAR DE GRAN CANARIA \\ ${ }^{(3)}$ Médico Adjunto. Coordinador Unidad de Mano, Miembro Superior y Nervio Periférico. \\ Hospital Universitario NuEstra SEÑoRa de la CANDELARIa. TENERIFE \\ ${ }^{(4)}$ Profesor Colaborador Universidad de Las Palmas de Gran Canaria
}

Correspondencia:

Dr. Alberto Marcos García

Hospital Universitario Insular de Gran Canaria. Servicio de Cirugía Ortopédica y Traumatología

Avenida Marítima del Sur, s/n

35016 Las Palmas de Gran Canaria

Tels.: 928441901 - 928441902

e-mail: amarcosg@comlp.es

Introducción: Los tumores glómicos son tumores benignos, poco frecuentes, localizados preferentemente en la mano, que presentan una clínica muy característica y cuyo tratamiento es quirúrgico.

Material y métodos: Se han revisado retrospectivamente 75 tumores glómicos periungueales, diagnosticados clínicamente y confirmados por estudio anatomopatológico que fueron extirpados quirúrgicamente por vía lateroungueal.

Resultados: El empleo de la vía lateroungueal para la exéresis de los tumores glómicos periungueales fue efectiva en todos los casos, sin originar distrofias ungueales y con un porcentaje de recidivas bajo $(11,9 \%)$.

Discusión: El diagnóstico de los tumores glómicos es fundamentalmente clínico, aunque se apoya en las pruebas de imagen (radiografía, ecografía y resonancia magnética). Su tratamiento es quirúrgico, existiendo múltiples vías de abordaje. Existe un riesgo de recidiva, que debe ser conocido por el paciente previamente a la cirugía.

Conclusiones: El acceso lateroungueal en los tumores glómicos es un método seguro, que permite su exéresis completa.

Palabras clave: tumor glómico, mano.
Introduction: Glomus tumors are benign tumors, rare, located most often in the hands, which show a very characteristic clinical and whose treatment is surgical.

Materials and methods: We have reviewed 75 periungual glomus tumors retrospectively, diagnosed clinically and confirmed by histological studies, that were surgically removed by lateroungueal approach.

Results: The use of the lateroungueal approach for resection of the periungual glomus tumors was effective in all cases, without causing new distrofias nail and with a low rate of recurrence $(11,9 \%)$.

Discussion: The diagnosis of glomus tumors is primarily clinical, but is supported by the evidence imaging ( $\mathrm{x}$-ray, ultrasound and magnetic resonance). Their treatment is surgical, multiple approach are described. There is a risk of recurrence, which should be known by the patient before surgery.

Conclusions: The approach lateroungueal in glomus tumors is a safe method, which allows complete resection.

Key words: Glomus tumor, hand. 


\section{INTRODUCCIÓN}

T

os tumores glómicos, descritos por Masson en $1924^{1}$, son tumores benignos y poco frecuentes (del 1 al $5 \%$ de todos los tumores de la mano $)^{2}$. Su origen parece ser los glomus neuromioarteriales ${ }^{2-5}$ responsables de la regulación del flujo sanguíneo y la temperatura $^{3}$. Se presentan más frecuentemente en mujeres, entre 20 y 40 años y su causa es desconocida $^{6}$. Su localización preferente es la mano $^{6,7}$, fundamentalmente en la falange distal de los dedos ${ }^{4,8}$. Se suelen presentar como una única lesión y excepcionalmente como formas múltiples $^{8}$. Se han publicado casos de afectación simultánea en dos dedos de una misma mano ${ }^{9}$ e incluso de presentación intraósea en la falange distal ${ }^{10}$. Su localización preferente es subungueal ${ }^{5,9}$.

La triada clínica clásica consiste en dolor local, dolor a la presión selectiva (test de Love), e hipersensibilidad al frío ${ }^{2}$. Suelen presentar una coloración azulada ${ }^{11}$, pérdida del color rojizo en el lecho ungueal, distrofias ungueales o fisuras longitudinales en la uña ${ }^{12}$. La prueba o test de Hildreth, que consiste en la colocación de un manguito de presión, por encima de la presión sistólica, a nivel proximal del brazo, con desaparición del dolor a la presión (negativización del test de Love), es altamente sugestiva de tumor glómico; de tal modo que incluso algunos autores sugieren su empleo en casos de dolor en el miembro superior de etiología poco clara ${ }^{2}$. No obstante, aunque esta clínica es muy característica, los tumores glómicos pueden presentar sintomatología poco específica $^{13}$, con cuadros de dolor que pueden asemejarse a otros procesos, lo que hace que el diagnóstico en estos casos sea, a menudo, tardío ${ }^{2,4,13,14}$. En la radiografía simple se pueden presentar como defectos óseos en la falange distal ${ }^{12}$.

Para facilitar su localización e incluso definir el tamaño de la lesión, se han empleado los ultrasonidos ${ }^{15}$, presentándose como áreas circunscritas hipoecoicas bajo la placa ungueal o la matriz ungueal ${ }^{12}$. La resonancia magnética (RM) y la angio-RM pueden asegurar el diagnóstico ${ }^{13},{ }^{16}$. También la RM puede ser útil en tumores glómicos ocultos, en pacientes con do- lor de causa poco clara ${ }^{17}$. La RM muestra masas bien delimitadas a nivel del lecho ungueal de baja o media señal en imágenes potenciadas en T1 y alta señal en imágenes potenciadas en $\mathrm{T} 2^{4,12,18}$.

Es preciso realizar diagnóstico diferencial con otros pequeños tumores de mano que pueden presentar esta triada clásica de dolor, dolor selectivo e hipersensibilidad al frío como puede ser el hemangioma subcutáneo ${ }^{19}$. También es preciso considerar el diagnóstico diferencial con los neuromas, las hiperplasias del corpúsculos del Pacini ${ }^{20,21}$ y con el fenómeno de Raynaud $^{13}$.

El tratamiento de los tumores glómicos es únicamente quirúrgico ${ }^{13,22,23}$. Se han descrito múltiples accesos para su extirpación: transungueal $^{5,14,23,24}$, transungueal triangular ${ }^{3}$, periungueal $^{24}$, bajo el lecho ungueal ${ }^{25}$, lateral ${ }^{26-28}$. Independientemente de la vía de acceso empleada, la escisión quirúrgica debe ser cuidadosa asegurando no lesionar las estructuras nerviosas $^{13}$ y reparando el lecho ungueal en el caso de las exéresis transungueales. Estos pequeños tumores glómicos de la mano son, a menudo, difíciles de extirpar y pueden presentar relativas altas tasas de recurrencia ${ }^{24,26}$, generalmente debidas a inadecuadas extirpaciones ${ }^{23,24,29}$. El conocimiento preoperatorio de su localización y tamaño facilita la extirpación y disminuye el riesgo de recidiva ${ }^{12,15}$.

Están descritas formas familiares de tumores glómicos. Estas formas hereditarias tienen un patrón autonómico dominante ${ }^{11,30,31}$.

Los tumores glómicos pueden también presentarse de forma múltiple, si bien son $\operatorname{raras}^{30}$, pues se han descrito menos de $200 \operatorname{casos}^{8}$. Suele tratarse de pequeñas lesiones angiomatosas que causan discreto dolor ${ }^{9} \mathrm{y}$ que pueden ser adquiridas o congénitas ${ }^{11}$.

Asimismo, están descritas formas malignas de los tumores glómicos denominados también glomangiosarcomas que son excepcionalmente $\operatorname{raras}^{32-34}$. El glomangiosarcoma es considerado un tumor de bajo grado de malignidad con tendencia a la recidiva local aunque han sido reflejadas metástasis a distancia, cuyo tratamiento, al igual que los tumores glómicos, es la escisión local ${ }^{32-34}$. 


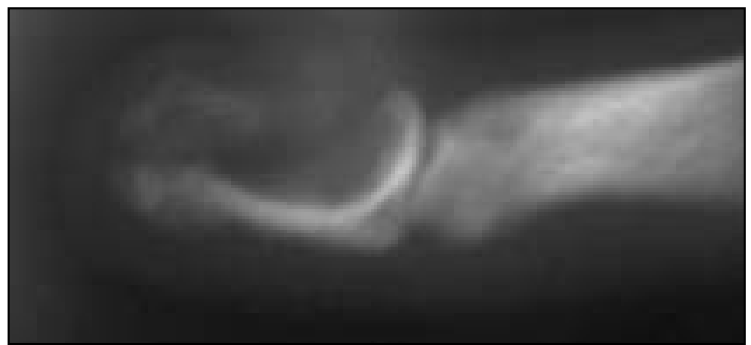

Figura 1: Radiografía que muestra erosiones en cara dorsal de falange distal.

\section{MATERIAL Y MÉTODO}

Se han revisado retrospectivamente 75 tumores glómicos periungueales, tratados consecutivamente, y confirmados histológicamente.

La edad media de presentación fue de 43 años (rango 21-70). Cincuenta y tres casos eran mujeres y 22 hombres, sin diferencias significativas en cuanto al lado (38 izquierdos, 37 derechos). El tiempo transcurrido desde el inicio de los síntomas hasta el diagnóstico oscilo desde los 6 meses a los 10 años. El periodo medio de seguimiento postquirúrgico fue de 6 años (rango 1-17)

El diagnóstico se basó en la presentación clínica: exacerbación del dolor con el frío, dolor selectivo a punta de lápiz (test de Love), la reducción o desaparición del dolor al colocar un manguito de tensión en la región proximal del brazo (prueba o test de Hildreth) y en los cambios de coloración de la piel.

Se practicó estudio radiológico simple en todos los casos (Figura 1) y estudio por RM en casos de sintomatología poco clara, recidivas o intervenciones previas (Figura 2).

Todos los tumores, primarios y recidivas, fueron intervenidos de modo ambulatorio, bajo anestesia locorregional (plexo axilar) y mangui-

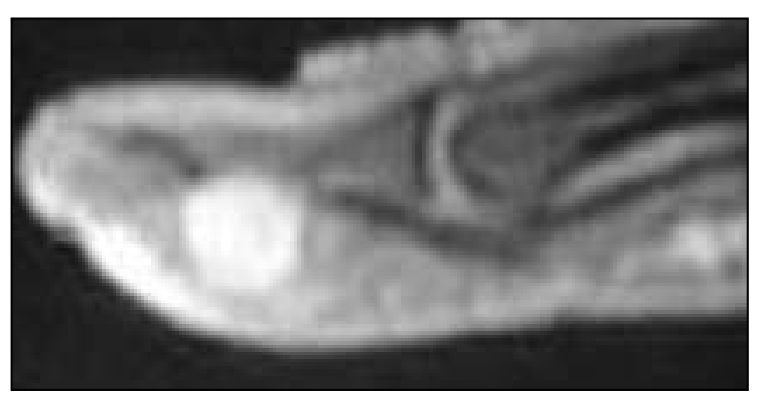

Figura 2: Masa bien delimitada en la RM.

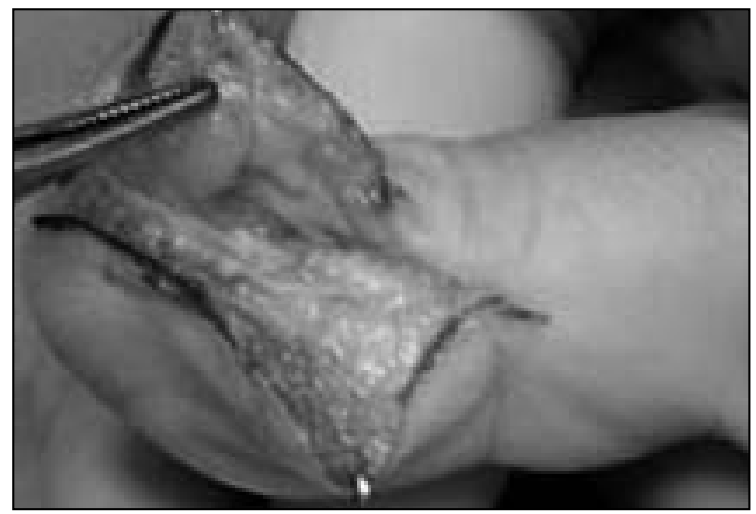

Figura 3: Acceso lateroungueal con levantamiento del complejo ungueal y localización del tumor glómico.

to de isquemia. Se realizó un acceso por vía lateroungueal, con levantamiento del complejo ungueal en los casos que fue preciso, que permitió la localización y la extirpación completa del tumor (Figuras 3, 4 y 5).

\section{RESULTADOS}

El dolor fue el síntoma principal en el 97\% de los casos. El diagnóstico clínico se basó en la exacerbación del dolor con el frío o con un golpe, la presencia del signo de Love y la positividad de la prueba o test de Hildreth en 63 casos. Además, en 30 casos, se apreciaba una mancha azulada en la piel. En las pruebas por imagen, 26 casos en la radiografía simple presentaban una impronta ósea en el área de localización del tumor. La RM se realizó en 36 de los casos, confirmando el diagnóstico en los mismos. La localización de los tumores fue: 42 estaban situados en el lecho ungueal, 21

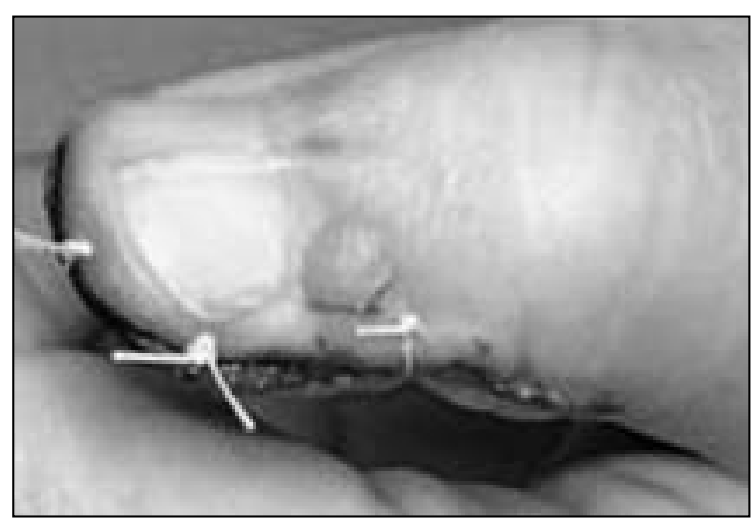

Figura 4: Cierre de la herida con exposición del tumor extirpado.

Revista Iberoamericana de Cirugía de la Mano 


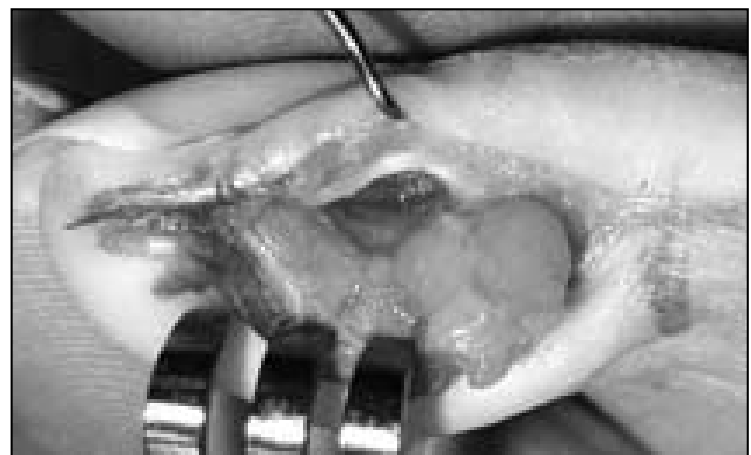

Figura 5: Acceso lateroungueal en la extirpación de un tumor glómico.

a nivel lateral de la uña y 12 en la base de la misma.

El empleo de la vía lateroungueal para la exéresis de estos tumores glómicos periungueales, permitió, en todos los casos, la visualización y resección del tumor. Aún así, se presentaron 14 recidivas, de las cuales 8 correspondían a nuestros equipos y 6 a tumores glómicos intervenidos previamente en otros centros, que fueron nuevamente intervenidas por esta misma vía, con exéresis completa del tumor y resolución del cuadro. La utilización de esta vía no originó ninguna distrofia ungueal, que no estuviera ya presente antes de la cirugía, ni ningún otro tipo de complicación reseñable.

\section{DISCUSIÓN}

Clásicamente, los tumores glómicos, se considera originados en el glomus neuromioarterial. A pesar de ello, trabajos como el de Abou Jaoude et al. ${ }^{35}$ cuestionan este origen. Los autores, en un estudio por inmunohistoquímica en una serie de 11 tumores glómicos, encontraron que las células tumorales eran positivas para anticuerpos antivimentin, negativos para anticuerpos anti-factor VIII e irregularmente positivos frente a anticuerpos anti-CD34. Con estos hallazgos, los autores afirmaban que no era posible confirmar la especificidad de los anticuerpos anti-CD34 para tumores glómicos en su serie, por lo que concluyeron que la hipótesis clásica del origen endotelial de los tumores glómicos, puede, probablemente, ser descartada ante la ausencia de expresión de estos dos anticuerpos anti-factor VIII y anti-CD34.

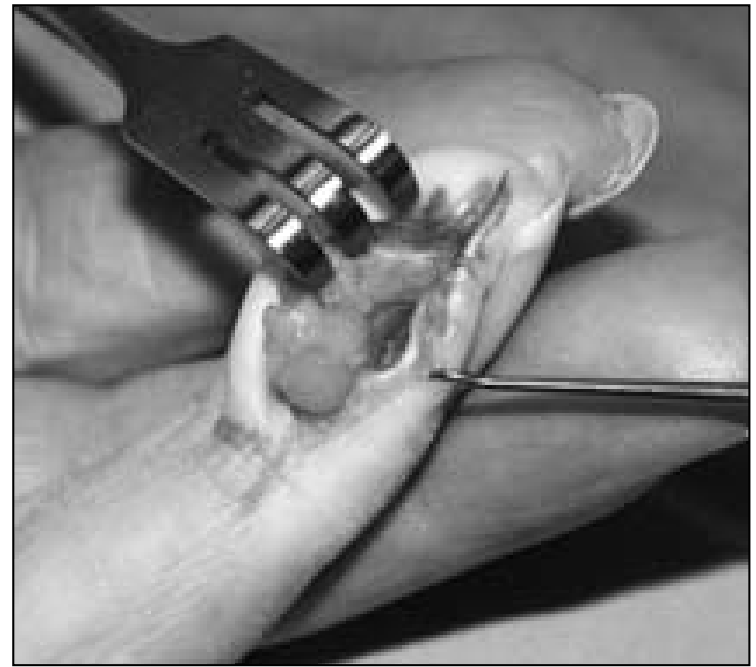

El diagnóstico del tumor glómico es fundamentalmente clínico, como se constata en todas las series revisadas (Tabla I). Así, el dolor a punta de lápiz, test de Love, presenta para Bhaskaranand y Navadgi ${ }^{36}$, en una serie de 18 casos, una sensibilidad del $100 \%$. Para la hipersensibilidad al frío, estos mismos autores, encontraron una sensibilidad del $100 \%$ y una especificidad del $100 \%$, mientras que en la prueba de Hildreth la sensibilidad fue del 77,4\% y la especificidad del $100 \%$. Giele ${ }^{37}$, en un estudio sobre el valor diagnóstico de la prueba de Hildreth encontró una sensibilidad del $92 \%$, con una especificidad del $91 \%$, un valor predictivo positivo del $92 \%$ y un valor predictivo negativo del $91 \%$.

Los cambios de coloración, generalmente azulados, son frecuentes en el área del tumor, en nuestra serie lo presentaron el $40 \%$ de los casos; en las diferentes series estos cambios de coloración están presentes entre el $29 \%$ de la

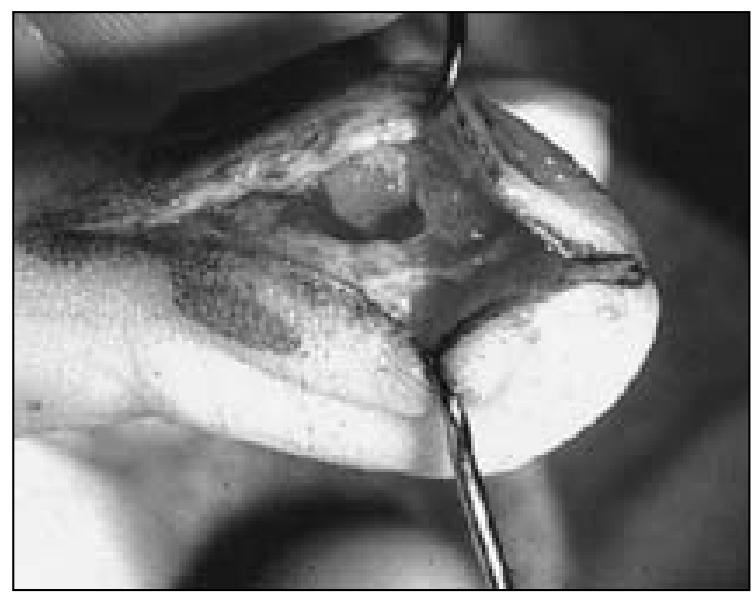


Tabla I - TUMORES ÓSEOS MALIGNOS

\begin{tabular}{|c|c|c|c|c|c|c|}
\hline & $\begin{array}{l}\text { Número } \\
\text { casos }\end{array}$ & $\begin{array}{l}\text { Diagnóstico } \\
\text { clínico }\end{array}$ & $\mathrm{Rx}(\%)$ & RM & Acceso & $\begin{array}{c}\text { Recidivas } \\
(\%)\end{array}$ \\
\hline Murthy et al. $2006^{5}$ & 2 & $\mathrm{Si}$ & 0 & 0 & Transungueal & 0 \\
\hline Moon et al. $2004^{38}$ & 16 & $\mathrm{Si}$ & - & - & Transungueal & 0 \\
\hline Vasisht et al. $2004^{26}$ & 19 & $\mathrm{Si}$ & - & - & Lateral & 15,7 \\
\hline Tomak et al $2003^{23}$ & 14 & $\mathrm{Si}$ & 28,5 & 0 & Transungueal & - \\
\hline Assmus y Dombert $2002^{1}$ & 36 & $\mathrm{Si}$ & - & 3 (duda diagnóstica) & Transungueal & 5,5 \\
\hline Takata et al. $2001^{24}$ & 30 & $\mathrm{Si}$ & - & - & Transungueal y periungueal & 30 \\
\hline Ekin et al. $1997^{25}$ & 9 & Sí (transiluminación) & - & - & Bajo el lecho ungueal & - \\
\hline Van Geertruyden et al. $1996^{1}$ & 1451 & $\mathrm{Si}$ & 36 & 0 & Transungueal & 3,9 \\
\hline Gandon et al. $1992^{28}$ & 48 & $\mathrm{Si}$ & 40 & Ocasional & Transungueal y lateral & 4,3 \\
\hline
\end{tabular}

serie de Van Geertruyden et al. ${ }^{14}$ y el $100 \%$ de la serie de Moon et al..$^{38}$. Ekin et al. ${ }^{25}$, en una serie de 9 tumores, describen el empleo de un test de transiluminación como método diagnóstico efectivo en estos tumores. Los hallazgos radiográficos de la radiografía simple son característicos en estos tumores $(34,6 \%$ de nuestra serie), pero no siempre están presentes, oscilan del $28,5 \%$ de la serie de Tomak et al. ${ }^{23}$ al $40 \%$ de la serie de Gandon et al. ${ }^{28}$ (Tabla I). Hay pocas referencias en la bibliografía al empleo de los ultrasonidos como método diagnóstico en los tumores glómicos ${ }^{12,15}$, pero pueden constituir una alternativa económica en la localización preoperatoria de estos tumores, aunque en nuestra serie no fueron empleados en el diagnóstico. Respecto a la RM, aunque en la bibliografía queda ampliamente reseñado que es un buen método para la localización de los tumores glómicos, presentando una buena correlación con los hallazgos quirúrgicos en cuanto a tamaño, localización y extensión ósea ${ }^{13}, 16,39$, creemos, al igual que otros autores ${ }^{3,17,27,28,40}$, que debe reservarse para aquellos casos con sintomatología poca clara, para las recidivas o en casos multioperados. Al-Quattam et al. ${ }^{41}$ ilustran este hecho en una serie de 42 casos de tumores glómicos, en los que la RM presentó una sensibilidad del $90 \%$, una especificidad del $50 \%$ un valor predictivo positivo del $97 \%$ y un valor predictivo negativo del 20\%; así los autores concluyen, que en función de estos resultados y del coste de la RM, desaconsejan el uso de la RM preoperatoria en pacientes clínicamente diagnosticados de tumor glómico.

En lo referente a la vía de acceso, mayoritariamente se ha venido empleando la vía transungueal (Tabla I), aunque también se emplea la vía periungueal para los tumores localizados en el área central o pulpejo ${ }^{24}$. Hemos encontrado tres series en las que se utilizó la vía lateral ${ }^{26-28}$ con resultados similares a nuestra serie $(11,9 \%$ de recidivas, 0 distrofias) en cuanto a resolución del cuadro y resultados estéticos (Tabla I).

La existencia de recidivas en las diferentes series oscila desde el $0 \%$ de la series de Baskaranand y Navdagi ${ }^{36}$ y Moon et al. ${ }^{38}$ al $30 \%$ de la serie de Takata et al. ${ }^{24}$ (Tabla I), encontrándose nuestra serie en unos valores medios del 18,6\%, aunque las recurrencias en los pacientes intervenidos primariamente en nuestro Servicio sería del $11,9 \%$. Este riesgo de recidivas debe llevar a informar de modo sistemático a todos los pacientes en el preoperatorio de esta complicación poco mencionada en la literatura $^{27}$.

El empleo de la vía lateral en nuestra serie no originó ninguna distrofia nueva. Esta com- 
plicación, aunque no se recoge en todas las series, si parece ser más mencionada en los abordajes transungueales, en los que a menudo es preciso reparar el lecho ungueal; Moon et al. ${ }^{38}$ aportan 3 casos de distrofia en una serie de $16(19 \%)$, Takata et al. ${ }^{24}$ presentan 5 distrofias en una serie de 30 casos $(16,6 \%)$. Por el contrario, ninguna de las series que utilizan el acceso lateral menciona distrofias ungueales tras la extirpación del tumor gló$\operatorname{mico}^{26-28}$.

\section{CONCLUSIÓN}

El acceso lateral de los tumores glómicos periungueales, con levantamiento de todo el complejo ungueal, en los casos de localización subungueal, es un método seguro y conduce a la exéresis completa del tumor sin distrofia, ni ningún otro tipo de complicación. Es necesario que los pacientes conozcan, previamente a la cirugía, el riesgo de recidiva de estos tumores.

\section{BIBLIOGRAFÍA}

1. Masson P. Le glomus neuromyo-artériel des régions tactiles et ses tumeurs. Lyon Chir, 1924; 21: 257-80.

2. Nebreda CL, Urban BJ, Taylor AE. Upper extremity pain of 10 years duration caused by a glomus tumor. Reg Anesth Pain Med, 2000; 25: 69-71.

3. Assmus H, Dombert T. Glomus tumours of the extremities: localisation and operative treatment in 36 cases. Handchir Mikrochir Plast Chir, 2002; 34: 103-7.

4. Shih TT, Sun JS, Hou SM, Huang $\mathrm{KM}$, Su TT. Magnetic resonance imaging of glomus tumour in the hand. Int Orthop, 1996; 20: 342-5.

5. Murthy PS, Rajagopal R, Kar PK, Grover S. Two cases of subungual glomus tumor. Indian $\mathrm{J}$ Dermatol Venereol Leprol, 2006; 72: 47-9.

6. Schiavone Panni A, Tulli A, lacopino C, Bucca C. Rare localization of a glomus tumor in the knee. Arch Putti Chir Organi Mov, 1991; 39: 55-61.

7. Kale SS, Rao VK, Bentz ML. Glomus tumor of the index finger. J Craniofac Surg, 2006; 17: 801-4.

8. D'Acri AM, Ramos-e-Silva M, Basilio-de-Oliveira $C$, et al. Multiple glomus tumors: recognition and diagnosis. Skinmed, 2002; 1: 94-8.

9. Graham B, Wolff TW. Synchronous subungual glomus tu- mours in adjacent digits. J Hand Surg B, 1992; 17: 575-6.

10. Settakorn J, Chalidapong P, Rangdaeng $S$, et al. Primary intraosseous glomus tumor: a case report. J Med Assoc Thai, 2001; 84: 1641-5.

11. Calduch L, Monteagudo C, Martinez-Ruiz E, et al. Familial generalized multiple glomangiomyoma: report of a new family, with immunohistochemical and ultrastructural studies and review of the literature. Pediatr Dermatol, 2002; 19: 402-8.

12. Takemura N, Fujii N, Tanaka T. Subungual glomus tumor diagnosis based on imaging. J Dermatol, 2006; 33: 389-93.

13. Monacelli G, Cascioli I, Mongardini $\mathrm{M}$, et al. Glomus tumor and neovascular syndrome of the arm: a clinical case. G Chir, 2003; 24: 235-8.

14. Van Geertruyden J, Lorea P, Goldschmidt D, et al. Glomus tumours of the hand. A retrospective study of 51 cases. J Hand Surg B, 1996; 21: 257-60.

15. Chen $\mathrm{SH}$, Chen $\mathrm{YL}$, Cheng $\mathrm{MH}$, et al. FC. The use of ultrasonography in preoperative localization of digital glomus tumors. Plast Reconstr Surg, 2003; 112: 115-9.

16. Boudghene FP, Gouny P, Tassart $M$, et al. Subungual glomus tumor: combined use of MRI and three-dimensional contrast
MR angiography. J Magn Reson Imaging, 1998; 8: 1326-8.

17. Matloub HS, Muoneke VN, Prevel $C D$, et al. Glomus tumor imaging: use of MRI for localization of occult lesions. J Hand Surg A, 1992; 17: 472-5.

18. Constantinesco A, Arbogast S, Foucher $G$, et al. Detection of glomus tumor of the finger by dedicated MRI at $0.1 \mathrm{~T}$. Magn Reson Imaging, 1994; 12: 1131-4.

19. Al-Qattan MM. A small cavernous vascular malformation in the hand mimicking a glomus tumour. J Hand Surg B, 1996; 21: 693-4.

20. Jones NF, Eadie P. Pacinian corpuscle hyperplasia in the hand. J Hand Surg A, 1991; 16: 865-9.

21. Greider JL Jr, Flatt AE. Glomus tumor associated with Pacinian hyperplasia - case report. J Hand Surg A, 1982; 7: 113-7.

22. Giberto M, Berretti B, Giua R, et al. A rare case of glomus tumor of the fingers. Chir Ital, 1983; 35: 270-5.

23. Tomak Y, Akcay I, Dabak N, Eroglu L. Subungual glomus tumours of the hand: diagnosis and treatment of 14 cases. Scand J Plast Reconstr Surg Hand Surg, 2003; 37: 121-4.

24. Takata H, Ikuta $Y$, Ishida O, Kimori K. Treatment of subungual glomus tumour. Hand Surg, 2001; 6: 25-7. 
25. Ekin A, Ozkan M, Kabaklioglu T. Subungual glomus tumours: a different approach to diagnosis and treatment. J Hand Surg B, 1997; 22: 228-9.

26. Vasisht B, Watson HK, Joseph E, Lionelli GT. Digital glomus tumors: a 29-year experience with a lateral subperiosteal approach. Plast Reconstr Surg, 2004; 114: 1486-9

27. Foucher G, Le Viet D, Dailiana Z, Pajardi G. Glomus tumor of the nail area. Apropos of a series of 55 patients Rev Chir Orthop, 1999; 85: 362-6.

28. Gandon F, Legaillard P, Brueton $\mathrm{R}$, et al. Forty-eight glomus tumours of the hand. Retrospective study and four-year follow-up. Ann Chir Main Memb Super, 1992; 11: 401-5.

29. Mansat M, Bonnevialle P, Gay R, Durroux R. Glomus tumors of the hand. Presentation of fourteen cases. Ann Chir Main, 1985; 4: 43-50.

30. Moor EV, Goldberg I, Westreich M. Multiple glomus tumor: a case report and review of the literature. Ann Plast Surg, 1999; 43: 436-8.

31. Brouillard P, Ghassibe $M, P e-$ nington $A$, et al. Four common glomulin mutations cause two thirds of glomuvenous malformations («familial glomangiomas»): evidence for a founder effect. J Med Genet, 2005; 42: e13.

32. Pérez de la Fuente $T$, Vega $C$, Gutiérrez Palacios A, et al. Glomangiosarcoma of the hypothenar eminence: a case report. Chir Main, 2005; 24: 199-202.

33. Park JH, Oh $\mathrm{SH}$, Yang $\mathrm{MH}$, Kim NI. Glomangiosarcoma of the hand: a case report and review of the literature. J Dermatol, 2003; 30: 827-33.

34. Wetherington RW, Lyle WG, Sangueza OP. Malignant glomus tumor of the thumb: a case report. J Hand Surg A, 1997; 22: 1098-102.

35. Abou Jaoude JF, Roula Farah A, Sargi Z, et al. Glomus tumors: report on eleven cases and a review of the literature. Chir Main, 2000; 19: 243-52.
36. Bhaskaranand K, Navadgi BC. Glomus tumour of the hand. J Hand Surg B, 2002; 27: 229-31.

37. Giele H. Hildreth's test is a reliable clinical sign for the diagnosis of glomus tumours. J Hand Surg B, 2002; 27: 157-8.

38. Moon SE, Won JH, Kwon OS, Kim JA. Subungual glomus tumor: clinical manifestations and outcome of surgical treatment. J Dermatol, 2004; 31: 993-7.

39. Dupuis P, Pigeau I, Ebelin M, et al. The contribution of MRI in the study of glomus tumors Ann Chir Main, 1994; 13: 358-62.

40. Dailiana ZH, Drape JL, Le Viet D. A glomus tumour with four recurrences. J Hand Surg B, 1999; 24: 131-2.

41. Al-Qattan MM, Al-Namla A, AlThunayan A, et al. Magnetic resonance imaging in the diagnosis of glomus tumours of the hand. J Hand Surg B, 2005; 30: 535-40. 